\title{
New Types of Singularity in General Relativity: The General Cylindrically Symmetric Stationary Dust Solution
}

\author{
A. R. King \\ Department of Mathematics, University College London, London, U.K.
}

Received December 3, 1973

\begin{abstract}
The general solution of Einstein's equations for a stationary cylindrically symmetric distribution of pressure-free matter is obtained. It contains a function which may be freely prescribed. Using this freedom examples are given of new types of singularity in General Relativity.
\end{abstract}

\section{Introduction}

Recent work [1,2] on the problem of singularities in relativistic cosmological models has made it clear that a variety of types of singularity may occur, such as Ricci curvature ("big bang") singularities and intermediate singularities ("whimpers"). In [1] further types were discussed, including oscillating Ricci singularities and Weyl (conformal) singularities, but no exact examples could be given. We aim here to provide such examples, so we begin by making precise the notions of "oscillating Ricci" and "Weyl" singularities.

In an inextensible spacetime the existence of a singularity is deduced from that of an inextensible geodesic (or more generally an inextensible curve) having finite length as measured by a generalised affine parameter. Let the curve be $\chi(v)$, defined for $0 \leqq v \leqq v_{+}$, and suppose it cannot be extended beyond $v_{+}$. Consider the components $R_{a b c d}(v)$ of the Riemann tensor in orthonormal bases $\left\{\boldsymbol{e}_{a}\right\}$ defined along $\chi(v)$. Both oscillating Ricci singularities and Weyl singularities are types of curvature singularities: these occur when there is no orthonormal basis defined along $\chi(v)$ such that all $R_{a b c d}(v)$ tend to finite limits as $v \rightarrow v_{+}$. The division into Ricci and Weyl singularities comes from considering which part of the Riemann tensor is failing to tend to a limit. If the Ricci tensor components do not tend to finite limits in any orthonormal frame we have a Ricci singularity, and this is the familiar "big bang" singularity if some components actually diverge in every such frame. If a Ricci singularity is such that in some orthonormal frames some components $R_{a b}(v)$ oscillate finitely (but do not tend to a limit) and the rest do tend 
to limits, while in all other orthonormal frames at least some $R_{a b}(v)$ diverge, we call it an oscillating Ricci singularity.

Similarly, if the components $C_{a b c d}(v)$ of the Weyl tensor do not tend to finite limits in any orthonormal frame, but the Ricci tensor components do tend to finite limits in at least one such frame, we say we have a Weyl singularity.

(In fact the two examples we shall give will be cases where scalar polynomials of the relevant tensor (such as $R_{a b} R^{a b}$ ) do not tend to limits; this is clearly sufficient to show that some components of the tensor do not have limits in any frame. It may moreover be shown [1] to be also necessary in the case where the source of the gravitational field is a perfect fluid.)

Two further types of singularity which are not curvature singularities should be mentioned: first, if the $R_{a b c d}(v)$ go to finite limits in some orthonormal frame along $\chi(v)$ but not in any such frame parallely propagated along $\chi(v)$ we have an intermediate singularity ("whimper"). (See [1].) If all the $R_{a b c d}(v)$ have finite limits in some parallely propagated orthonormal frame as $v \rightarrow v_{+}$this is a locally extensible singularity: by a theorem of Clarke [3] there exists an open neighbourhood of $\chi(v)$ which can be extended in such a way that $\chi(v)$ can be continued beyond $\chi\left(v_{+}\right)$in this local extension. Cone singularities, covering-space singularities and the Taub-NUT singularity are examples of this type [4].

The question naturally arises as to whether there exist solutions of the Einstein field equations for a fluid-filled spacetime which exhibit such singularities. We shall prove this for oscillating Ricci and Weyl singularities by giving explicit examples.

We consider in fact stationary dust-filled spacetimes possessing cylindrical symmetry. Several members of this family are known exactly $[5,6]$ and our reason for considering it is that, as was first remarked by Maitra [6], the field equations reduce to an underdetermined system of ordinary differential equations. This can easily be understood using the result [7] that the vacuum field inside an infinitely long rotating cylinder of matter is simply flat space. This is the cylindrical analogue of Birkhoff's theorem, and just as Birkhoff's theorem shows that concentric spherical shells of matter do not interact, therefore allowing one to specify a "density law" arbitrarily, so here coaxial cylindrical shells do not interact, and we may specify arbitrarily either the density law, or a vorticity or shear law. The fluid lines (integral curves of the fluid 4-velocity $u^{a}$ ) are inextensible and incomplete if the vorticity $\omega(r)$ and shear $\sigma(r)$ do not go to finite limits for some finite value $r_{+}$of the proper radial coordinate $r$, so this will give us a singularity. This will not be a Ricci singularity if the density $\varrho(r)$ has a finite limit at $r_{+}$. Using the underdetermined nature of the field equations we are able to arrange 
that we get Weyl singularities in some cases. Similarly we can arrange that $\varrho(r)$ oscillates but everything else goes to finite limits, implying an oscillating Ricci singularity.

\section{The Field Equations}

Following Ellis (e.g. [8]) we choose an orthonormal basis $\left\{\boldsymbol{e}_{a}\right\}$ of vectors with $\boldsymbol{e}_{0}=\boldsymbol{u}$, normalised so that $\boldsymbol{e}_{a} \cdot \boldsymbol{e}_{b}=g_{a b}=\operatorname{diag}(-1,+1,+1,+1)$ and define the $\gamma_{b c}^{a}$ by the commutation relations $\left[\boldsymbol{e}_{a}, \boldsymbol{e}_{b}\right]=\gamma_{a b}^{c} \boldsymbol{e}_{c}$. A stationary, cylindrically symmetric spacetime admits a three-parameter Abelian group of motions acting on timelike hypersurfaces of transitivity. The vector $\boldsymbol{u}$ is tangent to these surfaces (since otherwise we would have a "tilted" Bianchi I dust universe, which is forbidden [9]) so without loss of generality we may take the normal $\boldsymbol{n}$ to the surfaces to be $\boldsymbol{e}_{3}$. As shown in [10] the vectors $\boldsymbol{e}_{A}$ (capital letters run from 0 to 2) generate the group reciprocal to the group of motions, and since the latter is Abelian so is its reciprocal group, giving

$$
\gamma_{A B}^{a}=0
$$

Since the surfaces orthogonal to $\boldsymbol{n}=\boldsymbol{e}_{3}$ are homogeneous $\boldsymbol{n}$ is geodesic. Using the relations $n_{a ; b}=\partial_{b} n_{a}-n_{f} \Gamma_{b a}^{f}, \Gamma_{a b c}=\frac{1}{2}\left(\gamma_{a b c}+\gamma_{c a b}-\gamma_{b c a}\right)$ (where $\partial_{a}(f)=e_{a}^{i} f_{; i}$ etc.) which follow [8] easily from the definitions, we find that

$$
\gamma_{A 3}^{3}=-n_{A ; b} n^{b}=0
$$

The only non-zero $\gamma_{b c}^{a}$ are now those of the form $\gamma_{B 3}^{A}\left(=-\gamma_{3 B}^{A}\right)$. Since the pressure in the fluid is zero, the Bianchi identities imply $\dot{u}_{a} \equiv u_{a ; b} u^{b}=0$, and from a similar computation to that giving (2.2) we find

$$
\gamma_{03}^{0} \equiv \dot{u}_{3}=0, \quad\left(\dot{u}_{1} \equiv \gamma_{01}^{0}=\dot{u}_{2} \equiv \gamma_{02}^{0}=0\right) \text {. }
$$

We find easily that the vorticity vector $\omega^{a}=\frac{1}{2} \eta^{a b c d} u_{b} u_{c ; d}$ has components

$$
\omega^{1}=-\frac{1}{2} \gamma_{23}^{0}, \quad \omega^{2}=\frac{1}{2} \gamma_{13}^{0}, \quad \omega^{3}=\omega^{0}=0 .
$$

We have still available the freedom to rotate $\boldsymbol{e}_{1}, \boldsymbol{e}_{2}$ in their 2 -space ${ }^{1}$, and we choose to align $e_{1}$ along $\omega$ at all points. Thus

$$
\omega^{2}=\gamma_{13}^{0}=0 \text {. }
$$

1 Which is actually a 2-surface, since they commute. 
Defining the shear tensor $\sigma_{a b}=u_{(a ; b)}-\frac{1}{3} u_{; c}^{c}\left(g_{a b}+u_{a} u_{b}\right)$ and collecting our results we find the only non-zero $\gamma_{b c}^{a}$ and $\sigma_{a b}$ are

$$
\left.\begin{array}{l}
\gamma_{23}^{0}=-2 \omega \quad\left(\omega \equiv \omega^{1}\right) \\
\gamma_{03}^{1}=-2 \sigma_{13}, \quad \gamma_{03}^{2}=-2 \sigma_{23} \\
\gamma_{13}^{1}, \quad \gamma_{23}^{2}, \quad \gamma_{23}^{1}, \quad \gamma_{13}^{2}
\end{array}\right\}
$$

(the fluid expansion $u_{; a}^{a}$ vanishes identically).

Our specialisations of the tetrad now make it possible to use the forms of the field equations and Jacobi identities given in the Appendix of [8]. The homogeneity gives $\partial_{0} \equiv \partial_{1} \equiv \partial_{2}=0$, and we can choose coordinates $\left(x^{A}, r\right)$ such that $\boldsymbol{e}_{3}=\boldsymbol{n}=\partial / \partial r$, the surfaces of transitivity being $\{r=$ constant $\}$. All variables are then functions of $r$ only. $[r$ is the proper distance along the spacelike integral curves of $\boldsymbol{n}$, and is clearly not the intrinsically defined radial coordinate $R$, which in general is some function $R(r)$.] The Jacobi identities are all identically satisfied and the (00), (01) field equations are

$$
\left.\begin{array}{rl}
\frac{1}{2} \varrho & =2 \omega^{2}-2 \sigma_{13}^{2}-2 \sigma_{23}^{2} \\
0 & =\frac{d \sigma_{13}}{d r}+\omega \gamma_{23}^{1}+\sigma_{23} \gamma_{13}^{2}+\sigma_{13}\left(\gamma_{23}^{2}+2 \gamma_{13}^{1}\right)
\end{array}\right\}
$$

(we take the cosmological constant $\Lambda=0$ ).

To characterise our solutions as representing infinite rotating cylinders we must require also that the reflections

$$
\begin{aligned}
& \mathscr{R}_{1}:\left(\boldsymbol{e}_{0}, \boldsymbol{e}_{1}, \boldsymbol{e}_{2}, \boldsymbol{e}_{3}\right) \rightarrow\left(\boldsymbol{e}_{0} ;-\boldsymbol{e}_{1}, \boldsymbol{e}_{2}, \boldsymbol{e}_{3}\right) \\
& \mathscr{S}_{1}:\left(\boldsymbol{e}_{0}, \boldsymbol{e}_{1}, \boldsymbol{e}_{2}, \boldsymbol{e}_{3}\right) \rightarrow\left(-\boldsymbol{e}_{0}, \boldsymbol{e}_{1},-\boldsymbol{e}_{2}, \boldsymbol{e}_{3}\right)
\end{aligned}
$$

should be isometries. (These correspond to the reflections $z \rightarrow-z$, and $(\varphi, t) \rightarrow(-\varphi,-t)$, of the usual cylindrical polar coordinates $(r, \varphi, z, t)$. $\mathscr{R}_{1}, \mathscr{S}_{1}$ will be isometries if [11]

(a) They are automorphisms of the Lie algebra of the $\boldsymbol{e}_{A}$.

(b) The second fundamental form $\tilde{\theta}_{a b}=\tilde{h}_{a}^{c} \tilde{h}_{b}{ }^{d} n_{c ; d}$ of the surfaces $\{r=$ constant $\}$ spanned by the $e_{A}$ is invariant under them $\left(\tilde{h}_{a b}=g_{a b}\right.$ $+n_{a} n_{b}$ ). Condition (a) is automatically satisfied, and applying (b) in the case of $\mathscr{R}_{1}$ implies $\tilde{\theta}_{01}=\tilde{\theta}_{12}=0$. We get no new conditions from $\mathscr{S}_{1}$. Now

and

$$
2 \tilde{\theta}_{01}=-\gamma_{31}^{0}-\gamma_{30}^{1}=-2 \sigma_{13}
$$

$$
2 \tilde{\theta}_{12}=\gamma_{23}^{1}+\gamma_{13}^{2} \text {. }
$$

Thus $\sigma_{13}=\gamma_{23}^{1}+\gamma_{13}^{2}=0$. From the second of (2.6),

$$
0=\left(\omega-\sigma_{23}\right) \gamma_{23}^{1} .
$$

Hence either $\omega=\sigma_{23}, \sigma_{13}=0$ or $\gamma_{23}^{1}=\gamma_{13}^{2}=\sigma_{13}=0$. 
Now from the first of (2.6) $\omega=\sigma_{23}, \sigma_{13}=0$ implies $\varrho=0$ so for non-vacuum solutions we take

$$
\gamma_{23}^{1}=\gamma_{13}^{2}=\sigma_{13}=0 .
$$

Writing now $\sigma_{23}=\sigma, \gamma_{13}^{1}=\gamma_{1}, \gamma_{23}^{2}=\gamma_{2}$ the field equations reduce to

$$
\begin{aligned}
\varrho & =4\left(\omega^{2}-\sigma^{2}\right) \\
0 & =\frac{d}{d r}(\sigma-\omega)+(\sigma-\omega) \gamma_{1}+2 \gamma_{2} \sigma \\
-\frac{1}{2} \varrho & =\frac{d \gamma_{1}}{d r}+\gamma_{1}\left(\gamma_{1}+\gamma_{2}\right) \\
0 & =\frac{d \gamma_{2}}{d r}+\gamma_{2}\left(\gamma_{1}+\gamma_{2}\right)
\end{aligned}
$$

and

$$
0=\gamma_{1} \gamma_{2}+(\omega-\sigma)^{2}
$$

where the last equation is a linear combination of (00), (11), (22), (33). It is easy to show that it is a first integral of the other equations, so we drop now (02) from our system. We write

$$
\omega=A \cosh \lambda, \quad \sigma=A \sinh \lambda .
$$

where $A, \lambda$ are (possibly complex) functions of $r$, and write also

$$
\gamma_{1}+\gamma_{2}=\Gamma
$$

Then the equations become simply

$$
\left.\begin{array}{rl}
\varrho & =4 A^{2} \\
\frac{d \Gamma}{d r}+\Gamma^{2} & =-2 A^{2} \\
\frac{d \gamma_{2}}{d r}+\Gamma \gamma_{2} & =0 \\
\gamma_{1} \gamma_{2} & =-A^{2} e^{-2 \lambda}
\end{array}\right\}
$$

where the second equation is $(11)+(22)$. Writing $\alpha=\left(\gamma_{2}\right)^{-1}$ we can express all quantities in terms of $\alpha$ and its derivatives:

$$
\begin{gathered}
\gamma_{2}=\alpha^{-1}, \quad \Gamma=\alpha^{-1} \alpha^{\prime}, \quad \gamma_{1}=\alpha^{-1}\left(\alpha^{\prime}-1\right) \\
\varrho=4 A^{2}=-\frac{2 \alpha^{\prime \prime}}{\alpha}, \quad e^{2 \lambda}=\frac{\alpha \alpha^{\prime \prime}}{2\left(\alpha^{\prime}-1\right)}=\frac{A^{2} \alpha^{2}}{1-\alpha^{\prime}}
\end{gathered}
$$

where

$$
\alpha^{\prime}=d \alpha / d r, \quad \alpha^{\prime \prime}=d^{2} \alpha / d r^{2} \text {. }
$$


This makes explicit the underdetermined nature of the solutions: we may choose $\alpha$ arbitrarily, subject only to the constraints that $A^{2}$ and $e^{2 \lambda}$ are positive for some range of $r$. The resulting solutions are invariantly distinguished from one another by the differing values of $\varrho=4 A^{2}$.

\section{Global Properties}

We are chiefly interested in the singularities of solutions given by various choices of $\alpha$ in (2.11). In particular we shall require the components of the Weyl tensor $C_{a b c d}$, so we compute its "electric" and "magnetic" parts, $E_{a c}=C_{a b c d} u^{b} u^{c}, H_{a c}=\frac{1}{2} \eta_{a b}{ }^{g h} C_{g h c d} u^{c} u^{d}$. Their nonvanishing components are

$$
\left.\begin{array}{l}
E_{11}=\frac{2}{3}\left(\sigma^{2}-\omega^{2}\right), \quad E_{22}=\frac{5}{3} \sigma^{2}+\frac{\omega^{2}}{3}-2 \omega \sigma \\
E_{33}=-\frac{7}{3} \sigma^{2}+\frac{\omega^{2}}{3}+2 \omega \sigma, \quad H_{13}=\gamma_{1}(\sigma-\omega)
\end{array}\right\}
$$

These determine the Weyl tensor completely, since

where

$$
\begin{aligned}
C_{a b c d}= & \left(\eta_{a b p q} \eta_{c d r s}+g_{a b p i} g_{c d r s}\right) u^{p} u^{r} E^{q s} \\
& -\left(\eta_{a b p q} g_{c d r s}+g_{a b p q} \eta_{c d r s}\right) u^{p} u^{r} H^{q s}
\end{aligned}
$$

$$
g_{a b c d}=g_{a c} g_{b d}-g_{a d} g_{b c} \text {. }
$$

It follows from (2.11), (3.1) that if $\alpha \rightarrow 0$ and $\sigma, \omega$ are well behaved, $H_{13} \rightarrow \infty$ in general. However, from the fact that

$$
\tilde{\theta}_{11}=\gamma_{1}=\alpha^{-1}\left(\alpha^{\prime}-1\right), \quad \tilde{\theta}_{22}=\gamma_{2}=\alpha^{-1}, \quad \tilde{\theta}_{33}=0
$$

it is clear that what is causing $H_{13}$ to be badly behaved is the focussing of the radial geodesics tangent to $\boldsymbol{n}$ for some value of the proper distance $r$ along them, which will occur at any axes of symmetry of our solutions. In general any such "axis" will be singular, for in view of the fact that $\boldsymbol{u}$ is the unique (since $\varrho>0$ ) timelike eigenvector of the Ricci tensor the divergence of $H_{13}$ and hence $H_{a b} H^{a b}$ implies the divergence of a Riemann tensor scalar polynomial. Our tetrad basis is always singular where $\alpha=0$, but in some cases we may interpret this as the usual removable singularity $r=0$ of cylindrical polar coordinates. From (2.11), (3.1) we have

$$
H_{13}=\alpha^{-2}\left(1-\alpha^{\prime}\right)^{3 / 2}
$$

and it follows easily that for $H_{13}$ to be finite at $r=0$ we must have $\alpha=r+(\mathrm{O})\left(r^{3}\right)$ near $r \ldots 0$. This will fit in with the condition that $\gamma_{2}$ must be an odd function of $r$ derived below.) 
To be able to interpret the singularity $\alpha=0$ as an axis we require certain extra conditions: first, we want to be able to identify all points $(t,-r, \varphi, z)$ with $(t, r, \varphi+\pi, z)$, so $\varrho, \omega, \sigma$ must be even functions of $r$ and $\gamma_{1}, \gamma_{2}$ must be odd functions of $r$. (The radial geodesics converge $\left(\tilde{\theta}_{a}^{a} \rightarrow-\infty\right.$ ) as $r \rightarrow 0$ from one side and expand out symmetrically $\left(\tilde{\theta}_{a}^{a} \rightarrow-+\infty\right)$ as $r$ passes through zero to the other side.) Secondly, to be able to identify points $(t, r, \varphi, z)$ with $(t, r, \varphi+2 n \pi, z), n=$ integer, we must have that the circumference of a small circle about the axis is equal to $2 \pi \times$ the radius (elementary flatness). Finally we would like to have no more than two such "axes", for this is the largest number of axes a spacetime can have if we demand that moving through an angle of $2 \pi$ about one axis gives a rotation of $2 \pi$ about the other axes (Hawking and Ellis, private communication). (Of course we can have any number of "local" axes, which do not obey this condition, as for example in LRS spaces [8].) To see if any intermediate singularities are occurring, we need to consider parallel propagated tetrads along various curves in the spacetime. (Although all Riemann tensor components may be finite in our frame, this might be infinitely Lorentz-rotated relative to a parallely propagated frame; Riemann tensor components in this frame would diverge, giving an intermediate singularity - see [1].) An obvious curve to consider is an integral curve of $\boldsymbol{n} ; v^{b}$ is parallel propagated along $n^{a}$ if $v_{; a}^{b} n^{a}=0$ i.e.

$$
\frac{d v^{b}}{d r}=-\Gamma_{3 f}^{b} v^{f}
$$

Using (2.5), (2.7) this gives $v^{1}=$ constant, $v^{3}=$ constant,

$$
\frac{d v^{0}}{d r}=-(\sigma+\omega) v^{2}, \quad \frac{d v^{2}}{d r}=-(\sigma+\omega) v^{0} .
$$

Thus if e.g. $v^{a}=v \delta_{2}^{a}$ at $r=r_{0}$ we find

where

$$
\left.\begin{array}{rl}
v^{a}(r) & =(v \sinh \beta, 0,0, v \cosh \beta(r)) \\
\beta & =-\int_{r_{0}}^{r}(\sigma+\omega) d r=-\int_{r_{0}}^{r} A e^{\lambda} d r .
\end{array}\right\}
$$

Thus a parallel propagated frame along an integral curve of $\boldsymbol{n}$ suffers a finite Lorentz rotation (boost) if the integral in (3.2) converges. This will certainly be so (for finite $r, r_{0}$ ) if $\sigma, \omega$ are bounded, and may even be so if they are not. Parallel propagation along the integral curves of the various $\boldsymbol{e}_{A}$ produces rotations at a constant rate of the parallel propagated basis relative to our basis. These therefore will always give finite Lorentz transformations for propagation through finite affine distances. Thus for the purpose of discovering whether the parallel propagated basis 
along a given curve suffers a finite or infinite Lorentz rotation we need only consider the component of the curve parallel to $e_{3}$, and the corresponding $\beta(r)$ in (3.2).

Given a solution (2.11) we may find coordinates $x^{i}$ (although we shall not actually do this for the solutions considered here) by letting $\boldsymbol{e}_{a}=e_{a}^{i} \partial / \partial x^{i}$ and choosing the $\boldsymbol{e}_{a}^{i}$ so that the commutation relations

$\left[\boldsymbol{e}_{A}, \boldsymbol{e}_{B}\right]=0,\left[\boldsymbol{e}_{0}, \boldsymbol{e}_{3}\right]=-2 \sigma \boldsymbol{e}_{2},\left[\boldsymbol{e}_{1}, \boldsymbol{e}_{3}\right]=\gamma_{1} \boldsymbol{e}_{1},\left[\boldsymbol{e}_{2}, \boldsymbol{e}_{3}\right]=-2 \omega \boldsymbol{e}_{0}+\gamma_{2} \boldsymbol{e}_{2}$

hold identically. We have already set $\boldsymbol{e}_{3}=\partial / \partial r$, and letting $\boldsymbol{e}_{1}=a(r) \partial / \partial z$ we find $a=\exp \left(-\int^{r} \gamma_{1} d r\right)$ for suitable choice of $z$.

We set

$$
\begin{aligned}
\boldsymbol{u}=\boldsymbol{e}_{0} & =b \partial / \partial t+c \partial / \partial \varphi \\
\boldsymbol{e}_{2} & =e \partial / \partial t+f \partial / \partial \varphi,
\end{aligned}
$$

where $b, c, e, f$ are functions of $r$ only. With this choice of coordinates the vectors $\partial / \partial t, \partial / \partial \varphi, \partial / \partial z$ are Killing vectors since they commute with all the $\boldsymbol{e}_{a}[10]$. The commutation relations are satisfied if

which imply

$$
\left.\begin{array}{l}
d b / d r=2 \sigma e, \quad d c / d r=2 \sigma f \\
d e / d r=2 \omega b-\gamma_{2} e, \quad d f / d r=2 \omega c-\gamma_{2} f
\end{array}\right\}
$$

$$
b f-c e=\exp \left(-\int^{r} \gamma_{2} d r\right)
$$

for suitable $t, \varphi$. The matrix $e_{a}^{i}$ has determinant

$$
(b f-c e) \exp \left(-\int^{r} \gamma_{1} d r\right)=\exp \left(-\int^{r}\left(\gamma_{1}+\gamma_{2}\right) d r\right)=\alpha^{-1}
$$

so the transformation between the $\boldsymbol{e}_{a}$ and the $x^{i}$ is non-singular for $\alpha \neq 0$ and singular when $\alpha=0$. Thus it is possible for $(t, r, \varphi, z)$ to be good coordinates even where the tetrad $\boldsymbol{e}_{a}$ is singular, i.e. on the "axis" $\alpha=0$. The coordinate metric $g_{i j}$ is given by

$$
g_{i j}=e_{i}^{a} e_{j}^{b} g_{a b}=-e_{i}^{0} e_{j}^{0}+e_{i}^{1} e_{j}^{1}+e_{i}^{2} e_{j}^{2}+e_{i}^{3} e_{j}^{3} .
$$

where $e_{i}^{a}$ is the inverse matrix to $e_{a}^{i}$. We find

$$
\begin{aligned}
d s^{2}= & \exp \left(2 \int^{r} \gamma_{2} d r\right) \\
& \cdot\left[-\left(f^{2}-c^{2}\right) d t^{2}+\left(b^{2}-e^{2}\right) d \varphi^{2}+2(f e-b c) d \varphi d t\right] \\
& +d r^{2}+\exp \left(2 \int^{r} \gamma_{1} d r\right) d z^{2}
\end{aligned}
$$


Coordinates are often used [6] in which the invariantly defined $(r, z)$ plane is given in conformally flat coordinates; to achieve this, let

where

$$
\left.\begin{array}{l}
\bar{r}=\int^{r} e^{-\Psi} d r \\
\Psi=\int^{r} \gamma_{1} d r .
\end{array}\right\}
$$

Then the last two terms in (3.4) become $e^{2 \Psi}\left(d \bar{r}^{2}+d z^{2}\right)$ as required. We may re-express the transformation (3.5) as

Using (2.11) we find

$$
\gamma_{1}=d \Psi / d r=e^{-\Psi} d \Psi / d \bar{r}
$$

so

$$
e^{-\Psi} d \alpha / d \bar{r}-\alpha e^{-\Psi} d \Psi / d \bar{r}=1
$$

$$
\alpha=\bar{r} e^{\Psi}
$$

with suitable choice of $\bar{r}$. (3.6) and the relation $\gamma_{2}=\alpha^{-1}$ imply now

$$
\exp \left(\int^{r} \gamma_{2} d r\right)=\exp \left(\int^{r} \frac{e^{-\Psi}}{\bar{r}} e^{\Psi} d \bar{r}\right)=\bar{r}
$$

From (3.4) we may also restate the "elementary flatness" conditions for $\alpha=0$ to be an axis; if $\varphi$ is our angular coordinate at $\alpha=r=0$ the circumference of a small circle is

$$
2 \pi \exp \left(\int^{r} \gamma_{2} d r\right)\left(b^{2}-e^{2}\right)^{\frac{1}{2}}
$$

This must equal $2 \pi \times$ the radius, which is $d r \sim r$, so the condition is

$$
\lim _{r \rightarrow 0}\left(b^{2}-e^{2}\right)^{\frac{1}{2}}=\lim _{r \rightarrow 0} r \exp \left(-\int^{r} \gamma_{2} d r\right)
$$

\section{Solutions}

We turn now to the discussion of exact solutions (2.11), discussing first the known solutions. The Ehlers-van Stockum solution [5] (see also [8]) are shear-free, so $\lambda=0$. From (2.11) this gives

$$
\alpha d^{2} \alpha / d r^{2}-2 d \alpha / d r+2=0,
$$

which can easily be solved using the coordinates (3.5), (3.6), leading to

$$
\Psi=k \bar{r}^{2}+l
$$

where $k, l$ are constants. Letting $k=-m^{2} / 8$ we quickly find $\varrho=4 \omega^{2}$ $=m^{2} \exp \left[\frac{m^{2} \bar{r}^{2}}{4}-2 l\right]$. Thus $\varrho, \omega$ become infinite as $\bar{r} \rightarrow \infty$. Since 
$r=\int e^{\Psi} d \bar{r}=\int \exp \left(\frac{-m^{2}}{8} \bar{r}^{2}+l\right) d \bar{r}$ we see that $r \rightarrow$ finite value as $\bar{r} \rightarrow \infty$. Thus, as remarked by Shepley [12] in connection with van Stockum's solution there is a Ricci curvature singularity at a finite proper distance from the axis of symmetry. The axis is certainly nonsingular since $\Psi$ is even in $\bar{r}$ (and hence $r$ ), and we find $b=$ constant, $e=b m \bar{r} / 2$, so the elementary flatness condition (3.8) is satisfied. $(\bar{r} \sim r$ as $r \rightarrow 0$ since $\Psi \rightarrow$ constant.)

We turn next to the geodesically complete solution discovered by Maitra [6]. This solution is everywhere analytic and contains no closed timelike lines. It is characterised by the fact that there is a timelike Killing vector of unit length. In Maitra's coordinates (3.5), (3.6) this solution is easily obtained by writing

$$
d \Psi / d \bar{r}=-y^{2} / 4 \bar{r} .
$$

Then using (2.11), (3.5), (3.6) it follows that

$$
\omega=\frac{e^{-\Psi}}{4}\left(\frac{d y}{d \bar{r}}+\frac{y}{\bar{r}}\right), \quad \sigma=\frac{e^{-\Psi}}{4}\left(\frac{d y}{d \bar{r}}-\frac{y}{\bar{r}}\right) .
$$

From (3.4) the timelike Killing vector $\partial / \partial t$ has unit length if

$$
\left(f^{2}-c^{2}\right) \exp \left(2 \int^{r} \gamma_{2} d r\right)=1
$$

Using (3.7) we therefore set

$$
f=\bar{r}^{-1} \cosh \delta(\bar{r}), \quad c=\bar{r}^{-1} \sinh \delta(\bar{r}) .
$$

Equations (3.3) [using (3.5) and the above expressions for $\omega$ and $\sigma$ ] now imply on eliminating $\delta(\bar{r})$

leading to

$$
y=\frac{1}{2}\left[\left(\frac{a^{2}}{\bar{r}^{2}}+4\right)-\frac{a}{\bar{r}}\right] \quad a=\mathrm{constant}
$$

$$
\Psi=-\frac{1}{4 x^{2}}\left[\left(1+x^{2}\right)^{\frac{1}{2}}-1\right]+\frac{1}{8}-\frac{1}{4} \log \frac{1}{2}\left[\left(1+x^{2}\right)^{\frac{1}{2}}+1\right]
$$

where $x=2 \bar{r} / a$, the value given by Maitra. The expressions for other quantities $\varrho, b, e$ etc. now follow easily and the conditions for a good axis are all satisfied. We give now some solutions which as far as the author is aware, are new.

We consider first solutions with

$$
\varrho=4 A^{2}=2 B^{2}=\text { constant . }
$$


From (2.11) we find that a solution is

$$
\alpha=B^{-1} \sin B r, \quad e^{2 \lambda}=\frac{2 \sin ^{2} B r}{1-\cos B r} .
$$

These solutions are clearly highly pathological, as we have an infinite number of candidates for "axes" $\alpha=0$. In fact this choice of $\alpha$ satisfies the regularity conditions for an axis at $r=0$, but the fact that $e^{2 \lambda} \rightarrow 0$ means that there is a Weyl singularity there. We see from (2.11) that $\omega \rightarrow \infty, \sigma \rightarrow-\infty$ with $\sigma \sim-\omega$. (3.1) shows that $E_{11} \rightarrow 0, E_{22} \sim 4 \omega^{2}$, $E_{33} \sim-4 \omega^{2}$ so

$$
E_{a b} E^{a b} \sim 32 \omega^{2} \rightarrow \infty
$$

Because all Ricci tensor components are either zero or proportional to $\varrho$, which is finite, this must be a Weyl singularity.

To give an example of an "oscillating Ricci" singularity, we consider solutions (2.11) with $\alpha$ of the form

$$
\alpha=e^{v}
$$

then

and

$$
\left.A^{2}=-\frac{1}{2}\left[\frac{d^{2} v}{d r^{2}}+\left(\frac{d v}{d r}\right)^{2}\right]\right]
$$

$$
e^{2 \lambda}=A^{2} e^{2 \lambda}\left[1-e^{v} \frac{d v}{d r}\right]^{-1} \cdot
$$

We try to find a suitable $v$ with

$$
\frac{d^{2} v}{d r^{2}}=-1-\sin ^{2} \frac{1}{r}
$$

We can choose our integration constant so that

$$
\frac{d v}{d r}=-r-\int_{0}^{r} \sin ^{2} \frac{1}{s} d s
$$

For $r>0, d v / d r$ is monotonically decreasing in $r$, and so $A^{2}$ (and hence $\varrho$ ) has the oscillatory character of the function $\sin ^{2} \frac{1}{r}$. We have also to check that $A^{2}, e^{2 \lambda}>0$ for $0 \leqq r \leqq$ some $r_{0}>0$. The above expression implies

$$
-r \geqq \frac{d v}{d r} \geqq-2 r \text {. }
$$

Thus we can choose a $v$ such that

and

$$
\frac{-r^{2}}{2} \geqq v \geqq-r^{2}
$$

$$
\left(\frac{d v}{d r}\right)^{2} \leqq 4 r^{2}
$$


From (4.1) this implies

where

$$
\begin{aligned}
& A^{2} \geqq \frac{1}{2}\left(1+\sin ^{2} \frac{1}{r}\right)-2 r^{2} \\
& e^{2 \lambda}=A^{2} e^{2 v}(1+k)^{-1}
\end{aligned}
$$

$$
k \geqq r e^{v} \geqq 0 \text {. }
$$

Thus for $0 \leqq r \leqq \frac{1}{2}$ both $A^{2}$ and $e^{2 \lambda}$ are strictly positive and finite, $\varrho, \omega, \sigma$ (and $\gamma_{1}, \gamma_{2}$ ) are finite and defined. However as $r \rightarrow 0, \varrho=4 A^{2}$ (which is a scalar polynomial: $\varrho^{2}=R_{a b} R^{a b}$ ) oscillates finitely and does not tend to a limit (it has the form $2 \sin ^{2} \frac{1}{r}+f(r)$ where $f$ is monotonic), so this is an "oscillating Ricci" singularity. This solution is again highly pathological, the density becoming negative for some $r$ between $\frac{1}{2}$ and 2 , and there being two "axes" $\alpha=0$ at $r= \pm \infty$.

We give finally a family of solutions which are non-pathological in the sense that they have a proper axis and their Riemann tensor components are always finite in a parallely-propagated frame.

We let

$$
\alpha=\int_{0}^{r} \frac{d s}{1+s^{2 n}} \quad n=\text { integer } \geqq 1
$$

then

and

$$
\left.\begin{array}{l}
A^{2}=n r^{2 n-1}\left(1+r^{2 n}\right)^{-2}\left[\int_{0}^{r} \frac{d s}{1+s^{2 n}}\right]^{-1} \\
e^{2 \lambda}=\frac{n}{r\left(1+r^{2 n}\right)} \int_{0}^{s} \frac{d s}{1+s^{2 n}} .
\end{array}\right\}
$$

Thus $e^{2 \lambda}, A^{2}>0$ for $r>0$ or $r<0$. At $r=0$ we have to investigate $\lim _{r \rightarrow 0} r^{-1} \int_{0}^{s} \frac{d s}{1+s^{2 n}}$. By l'Hôpital's Rule this is equal to $\lim _{r \rightarrow 0} \frac{1}{1+r^{2 n}}=1$. Hence $e^{2 \lambda} \rightarrow n$ and $A^{2} \rightarrow 1$ (if $n=1$ ) or 0 (if $n>1$ ) as $r \rightarrow 0$, and $A^{2}, e^{2 \lambda}$ are positive and bounded for all $r$, since

$$
\int_{0}^{r} \frac{d s}{1+s^{2 n}}<1+\int_{1}^{r} \frac{d s}{1+s^{2}}<1+\frac{\pi}{4} .
$$

As $r \rightarrow \infty, A^{2} \rightarrow 0$. We find

$$
\omega \sigma=\frac{1}{2}\left[\frac{n r^{n-1}}{\left(1+r^{2 n}\right)^{\frac{1}{2}}} \pm \frac{r^{n}}{\left(1+r^{2 n}\right)^{\frac{1}{2}} \int_{0}^{r} d s\left(1+s^{2 n}\right)^{-1}}\right]
$$

so as $r \rightarrow \infty, \omega$ and $\sigma$ tend to the same finite limit. The solutions are invariantly distinguished from Maitra's by the fact that $\varrho=\mathrm{O}\left(r^{-2 n-1}\right)$ 
as $r \rightarrow \infty$ whereas Maitra's $\varrho=\mathrm{O}\left(r^{-\frac{7}{4}}\right)$, and from the Ehlers-van Stockum solutions by the presence of shear. We must check that the "axis" $r=0$ is a good axis. First $\alpha=0$ only for $r=0$. Secondly, since $\alpha$ is an odd function, $\varrho, \sigma, \omega$ are even and $\gamma_{1}, \gamma_{2}$ are odd as required. Finally, to check the "elementary flatness" condition (3.8) we need the asymptotic form of $b, e$ and $\gamma_{2}$ as $r \rightarrow 0$.

Now

$$
\begin{aligned}
\alpha & =\int_{0}^{r} d s\left(1+s^{2 n}\right)^{-1}=\int_{0}^{r}\left[1-s^{2 n}+s^{4 n}-\cdots\right] d s \\
& =r\left[1-\frac{r^{2 n+1}}{2 n+1}+\frac{r^{4 n+1}}{4 n+1}-\cdots\right] .
\end{aligned}
$$

Thus

and hence

$$
\gamma_{2}=\alpha^{-1} \sim r^{-1}\left[1+\frac{r^{2 n+1}}{2 n+1}+\mathrm{O}\left(r^{4 n+1}\right)\right]
$$

$$
\exp \left(-\int^{r} \gamma_{2} d r\right) \sim \frac{1}{r} \exp \left[\frac{-r^{2 n+2}}{(2 n+1)(2 n+2)}+\mathrm{O}\left(r^{4 n+2}\right)\right]
$$

so

$$
\lim _{r \rightarrow 0} r \exp \left(-\int_{0}^{r} \gamma_{2} d r\right)=1
$$

From (3.8) the elementary flatness condition is then

$$
\lim _{r \rightarrow 0}\left(b^{2}-e^{2}\right)=1 .
$$

From (4.3) we have $\omega, \sigma \sim \frac{1}{2} n r^{n-1}$ for $n>1$, and $\omega \sim 1-\frac{3}{4} r^{2}, \sigma \sim-\frac{3}{4} r^{2}$ for $n=1$. Using (4.4) the Eq. (3.3) become

and

$$
\left.\begin{array}{rl}
d b / d r & =n r^{n-2}(e r) \\
d(e r) / d r & =n r^{n} b
\end{array}\right\} n>1
$$

$$
\left.\begin{array}{c}
d b / d r=-\frac{3}{2} r(e r) \\
d(e r) / d r=\left(2 r-\frac{3 r^{3}}{2}\right) b
\end{array}\right\} n=1 .
$$

These equations admit the solutions

$$
\left.\begin{array}{l}
\begin{array}{l}
b \\
= \\
e=n r^{n} /(n+1)+\mathrm{O}\left(r^{2 n}\right) \\
b
\end{array}=1-\frac{3}{8} r^{4}+\mathrm{O}\left(r^{6}\right) \\
e=r+\mathrm{O}\left(r^{3}\right)
\end{array}\right\} n=1 .
$$


Thus in all cases (4.5) is satisfied, and all solutions have a good axis. To see what happens to $R_{a b c d}$ in a parallely-propagated basis, we examine the integral $\beta(r)$ in (3.2); here this gives

$$
-\beta=\int_{r_{0}}^{r} n s^{n-1} /\left(1+s^{2 n}\right)^{\frac{3}{2}} d s
$$

which is bounded for all $r_{0}, r$ for consider the "worst" case $r_{0}=0, r=\infty$. Then $\beta<0$ and

$$
\begin{aligned}
-\beta & =\int_{0}^{1} \frac{n s^{n-1}}{\left(1+s^{2 n}\right)^{\frac{3}{2}}} d s+\int_{1}^{\infty} \frac{n s^{n-1}}{\left(1+s^{2 n}\right)^{\frac{3}{2}}} d s \\
& \leqq \int_{0}^{1} n d r+\int_{1}^{\infty} \frac{n s^{2 n-1}}{\left(1+s^{2 n}\right)^{\frac{3}{2}}} d s \\
& =n+\left[-\left(1+r^{2 n}\right)^{-\frac{1}{2}}\right]_{1}^{\infty}=n+\sqrt{2} .
\end{aligned}
$$

Hence all Riemann tensor components are finite (analytic!) in any parallel propagated orthonormal frame. This follows since all such frames, by the above result, suffer a finite Lorentz rotation relative to our frame, and all $R_{a b c d}$ are finite in our frame as $\varrho, \sigma, \omega$ are everywhere finite and $\gamma_{1}, \gamma_{2}$ are finite except on the axis $r=0$. Thus the only singularities that the family of solutions $\alpha=\int_{0}^{r} d s\left(1+s^{2 n}\right)^{-1}$ can have are locally extensible, and it is highly probable that in fact there are no singularities in these spacetimes. Unfortunately we are unable to confirm this by explicit examination of the geodesics because of the difficulty of solving exactly the system (3.3): this would give us the metric, or equivalently the Killing vector components in the tetrad basis, leading to constants of the motion. Similarly we are unable to check whether there are any closed timelike lines; a sufficient condition for their absence is $b^{2}-e^{2} \geqq 0$ for all $r$.

\section{Conclusion}

We have given a method of constructing all solutions of the field equations (with $\Lambda=0$ ) for stationary cylindrical symmetric distributions of pressure-free matter. Since the field equations give an underdetermined system in this case, there are arbitrarily many such solutions, and after showing how known solutions fit into our construction we have given some solutions which so far as the author is aware are new. Some of these were designed to exhibit new kinds of singularity not previously given in the literature. In all cases the matter is flowing past these singularities ( $\boldsymbol{u}$ lies in the surfaces $\{r=$ constant $\}$, and the singularities appear at particular values of $r$ ). This makes it seem highly likely that at least 
the Weyl singularities are unstable against degenerating into "big bang" singularities: should any matter be projected into them it seems certain that we would get $\varrho \rightarrow \infty$ here. Investigation of wave propagation in these spaces on the lines of [13] should help to resolve this point.

Acknowledgements. This work was carried out during the author's tenure of a U.K. Science Research Council Studentship at D.A.M.T.P., Cambridge, and he wishes to thank the members of the Relativity group at D.A.M.T.P. for many helpful comments.

\section{References}

1. Ellis, G.F.R., King,A.R.: Was the big bang a whimper? Preprint, Cambridge (1973)

2. Hawking, S.W., Ellis, G.F.R.: The large scale structure of space-time. Cambridge University Press (1973)

3. Clarke, C.J.S.: Local extensions in singular space-times. Preprint, Cambridge (1972)

4. Schmidt, B. G., Ellis, G.F.R.: Unpublished

5. Ehlers, J. : In: Royaumont Conference Volume (1959) van Stockum, W. J.: Proc. Roy. Soc. Edinburgh 57, 135 (1937) Wright, J.P.: J. Math. Phys. 6, 103 (1965)

6. Maitra, S. C.: J. Math. Phys. 7, 1025 (1966)

7. Davies, H., Caplan, T. A.: Proc. Cambridge Phil. Soc. 69, 325 (1971)

8. Ellis, G.F.R.: J. Math. Phys. 8, 1171 (1967)

9. King, A. R., Ellis, G.F.R.: Commun. math. Phys. 31, 209 (1973)

10. Ellis, G.F.R., MacCallum, M.A.H.: Commun. math. Phys. 12, 108 (1969)

11. Schmidt, B. G. : Commun. math. Phys. 15, 329 (1969)

12. Shepley, L. C.: Proc. Nat. Acad. Sci. U.S. 52, 1403 (1964)

13. King, A.R.: Scalar-wave perturbation of intermediate singularities. In: Ph.D.Dissertation, Cambridge University (1973) 
